\title{
Algoritmo RMLSA para redes ópticas flexibles en operación dinámica
}

\section{RMLSA algorithm for flexible optical networks under dynamic operation}

\author{
Ariel Leiva L. ${ }^{1 *} \quad$ Robert Ahumada C. ${ }^{2} \quad$ Alejandra Beghelli Z. ${ }^{3} \quad$ Ricardo Olivares V. $^{4}$ \\ Recibido 3 de mayo de 2016, aceptado 2 de agosto de 2017 \\ Received: May 3, 2016 Accepted: August 2, 2017
}

\begin{abstract}
RESUMEN
En este artículo se presenta un nuevo algoritmo de RMLSA (Routing, Modulation Level and Spectrum Allocation) para redes ópticas flexibles con operación dinámica en un sistema OFDM (Orthogonal Frequency Division Multiplexing). El algoritmo, denominado kSP-MAS-BRAF ( $k$-Shortest Path - Minimum Allocation of Subcarriers - Bit Rate Aware Fit), consiste en un algoritmo secuencial que primero define la ruta, luego el formato y nivel de modulación que minimiza el uso de espectro y, finalmente, la ubicación espectral que disminuye la probabilidad de bloqueo. El alcance óptico de la ruta es tomado en consideración para conformar la señal que usa un conjunto de subportadoras OFDM para transmitir la información.
\end{abstract}

Los resultados de simulación obtenidos muestran que, comparado con propuestas previas, kSP-MASBRAF obtiene probabilidades de bloqueo de hasta un orden de magnitud menor. Estos resultados son de interés para los operadores de telecomunicaciones que en un futuro reemplacen sus actuales redes DWDM (Dense Wavelength Division Multiplexing) por tecnologías del tipo dinámica y flexible.

Palabras clave: Algoritmos RMLSA, grilla flexible, redes ópticas de transporte, redes ópticas elásticas, modulación adaptiva.

\section{ABSTRACT}

A new RMLSA (Routing, Modulation Level and Spectrum Allocation) algorithm for OFDM dynamic flexible-grid optical networks is proposed. The algorithm, called kSP-MAS-BRAF (k-Shortest Path Minimum Allocation of Subcarriers - Bit Rate Aware Fit), is a sequential algorithm that first solves the routing problem, then selects the modulation format and level that minimize the use of spectrum and finally allocates spectrum trying to diminish the blocking probability. The optical reach of the route is taken into account to define how to establish the connection that uses a set of OFDM sub-carriers to transmit data.

Simulation results show that $\mathrm{kSP}-M A S-B R A F$ displays blocking probabilities up to one order of magnitude lower than previous proposals. These findings might be helpful for network operators studying the alternative of migrating, in a medium term, their networks to dynamic and flexible operation.

Keywords: RMLSA algorithms, flex grid, optical networks, optical transport networks, elastic optical networks, adaptive modulation.

1 Escuela de Ingeniería Eléctrica. Pontificia Universidad Católica de Valparaíso. Av. Brasil 2147, Valparaíso, Chile. E-mail: ariel.leiva@pucv.cl

2 Escuela de Ingeniería Eléctrica. Pontificia Universidad Católica de Valparaíso. Av. Brasil 2147, Valparaíso, Chile. E-mail: robert.ahumada.c@mail.pucv.cl

3 Facultad de Ingeniería y Ciencias. Universidad Adolfo Ibáñez. Av. Padre Hurtado 750, Viña del Mar, Chile. E-mail: alejandra.beghelli@uai.cl

4 Departamento de Electrónica. Universidad Técnica Federico Santa María. Av. España 1680, Valparaíso, Chile. E-mail: ricardo.olivares@usm.cl

* Autor de correspondencia. 


\section{INTRODUCCIÓN}

Actualmente, las redes de transporte DWDM (Dense Wavelength Division Multiplexing) representan la mejor alternativa para enfrentar el gran crecimiento de tráfico de datos entre países y ciudades [1]. La transmisión de datos en este tipo de redes se realiza mediante el envío de distintas señales de información que modulan, a altas tasas de transmisión digital, distintas portadoras ópticas (cada una en una longitud de onda específica) que se propagan simultáneamente por una misma fibra óptica. La longitud de onda de cada portadora está definida por la Recomendación G.694.1 [2] de la ITU (International Telecommunications Union), donde se establece la ubicación de cada portadora en el espectro asignado y la separación espectral entre ellas. A este tipo de sistemas se les conoce como sistemas DWDM de grilla espectral fija. La separación espectral más usada en sistemas de grilla fija es de $50 \mathrm{GHz}$, la que permite transmitir señales con velocidades de transmisión digital de 2, 5, 10 , 40 y 100 Gbps [3].
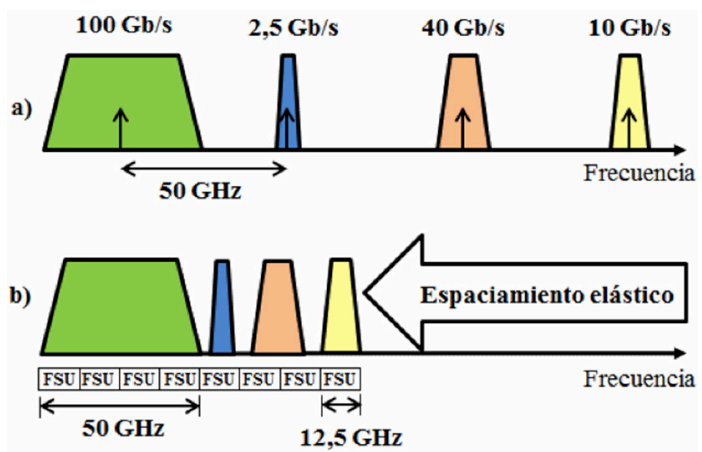

Figura 1. Espectro en un enlace usando un plan de frecuencia a) fijo y b) flexible.

La Figura 1 (a) muestra un ejemplo del uso del espectro de un enlace DWDM de grilla fija con 4 portadoras ópticas separadas por $50 \mathrm{GHz}$ y moduladas por señales a distintas velocidades de transmisión: 2, 5, 10, 40 y 100 Gbps.

La capacidad de las redes DWDM de grilla fija actuales es muy alta: experimentalmente se han reportado capacidades sobre $100 \mathrm{Tbps}$ por cada fibra óptica [4]. Sin embargo, el aumento exponencial del tráfico (con tasas anuales de 35 a 50\% [5]) y la creciente heterogeneidad del mismo (en el mediano plazo se esperan solicitudes de conexión a distintas tasas de transmisión digital desde 2,5 a $1.000 \mathrm{Gbps}$ [6]) amenazan con dejar obsoleto este tipo de operación. Por ejemplo, la separación de 50 $\mathrm{GHz}$ no es suficiente para implementar conexiones de 400 Gbps o superiores (que requieren anchos de banda mayores a $50 \mathrm{GHz}$ ). Para hacer viables estas conexiones sería necesario aumentar la separación espectral entre canales a $100 \mathrm{GHz}$. Sin embargo, dicha separación aumentaría la subutilización del espectro para señales menores a $100 \mathrm{Gbps}$ (que requieren anchos de banda menores a $100 \mathrm{GHz}$ [7]).

Una solución alternativa para acomodar conexiones de $400 \mathrm{Gbps}$ o velocidades superiores es la utilización flexible o elástica del espectro de una fibra óptica [8]. Esto es, la ubicación espectral de los distintos canales ópticos puede ser escogida sin ningún plan de frecuencias prefijado y el ancho de banda de cada uno de ellos es asignado de acuerdo con la tasa de transmisión digital y a la distancia a cubrir [9]. Este tipo de redes han sido nombradas como "redes ópticas flexibles" o "redes elásticas".

En la Figura 1 (b) se aprecia un conjunto de canales ópticos a los que se les asigna una ubicación y separación espectral no prefijada. De esta forma, la Figura 1 (b) ilustra cómo la asignación flexible del espectro permite acomodar las mismas 4 conexiones de la Figura 1 (a), pero con un uso más eficiente del espectro óptico mediante la disminución del espectro ocioso entre canales. Como consecuencia, se puede transmitir un mayor número de canales ópticos mitigando el problema del potencial crecimiento de tráfico de datos en el futuro cercano. Debido a sus ventajas, en términos de eficiencia de utilización del espectro, se espera que en los próximos años la asignación flexible de espectro deje obsoleto el plan de frecuencias fijo de la Rec. G.694.1 de la ITU.

Cabe notar que la asignación flexible de espectro tiene un nivel mínimo de granularidad conocido como FSU (Frecuency Slot Unit). Un FSU es la mínima porción de espectro asignable dentro de una fibra óptica (por ejemplo, 12,5 GHz). De esta forma, el espectro total de una fibra óptica y el asignable a cada conexión es un número discreto y múltiplo de FSUs. En la Figura 1 (b) se muestra el concepto de FSU, en donde las conexiones ópticas ocupan un número discreto de ellas. 
Existen dos grandes desafíos de implementación de las redes ópticas flexibles: por una parte, se requieren de dispositivos que funcionen de manera "flexible" [7]. Por otra parte, se deben diseñar algoritmos que encuentren, de manera rápida y eficiente, una trayectoria espectral (Spectrum Path [10]) disponible para atender una petición de conexión.

El primer desafío, fuera del alcance de este trabajo, corresponde al diseño y construcción de transpondedores, multiplexores y conmutadores ópticos capaces de ser configurados dinámicamente para generar, operar, y recibir señales ópticas en una longitud de onda central portadora a elección [11] o mediante un conjunto de subportadoras (como por ejemplo, a través del uso de OFDM: Orthogonal Frequency Division Multiplexing [12]), con distintas velocidades de transmisión digital (por ejemplo, de 2,5 Gbps a $1.000 \mathrm{Gbps}$ ) y distintos formatos de modulación (característica que determina el ancho de banda en conjunto a la velocidad de transmisión digital) de acuerdo con el alcance o distancia de la ruta de la conexión. En este trabajo se supone que la red ya cuenta con este tipo de dispositivos.

El segundo desafío, relacionado con la operación en redes flexibles, se conoce como el problema RMLSA (Routing, Modulation Level and Spectrum Allocation) [13]. Ante una solicitud de conexión caracterizada por los nodos a conectar y la velocidad de transmisión digital (bit rate) de la conexión a establecer, el problema RMLSA consiste en encontrar y asignar una ruta, un formato y/o nivel de modulación y una porción suficiente de espectro (misma posición espectral y número de FSUs contiguos en todos los enlaces de la ruta) para atender la solicitud de conexión. El foco de este artículo es proponer y evaluar una solución a esta problemática.

Para resolver el problema RMLSA se deben cumplir 5 restricciones: 1) Las FSUs asignadas a una conexión deben ser contiguas en el espectro (para conformar el canal óptico) [14], 2) se debe asignar el mismo grupo de FSUs en todos los enlaces de la ruta (restricción de continuidad para redes sin conversión de longitud de onda) [15], 3) una FSU no debe ser asignada a más de una conexión a la vez en una misma fibra óptica [16], 4) el alcance óptico de la señal óptica debe ser igual o mayor a la distancia de la ruta asignada [12], y 5) se debe asignar una banda de guarda entre los canales para evitar interferencias y facilitar la recuperación de las señales en el receptor [17].

De acuerdo con la operación de la red óptica, existen 2 maneras de resolver el problema RMLSA: estática y dinámica. En la estática, las conexiones se establecen permanentemente y la asignación de rutas, formato y/o nivel de modulación y espectro se resuelve offline con el principal objetivo de minimizar del uso de espectro [12]. En el caso dinámico, en cambio, las conexiones se establecen bajo demanda y los recursos de la red solo se reservan por el tiempo necesario para enviar información. Las soluciones RMLSA para operación dinámica suelen tener como objetivo disminuir la probabilidad de bloqueo de las conexiones [18]. Debido a que la operación dinámica se adapta mejor a cambios topológicos y de tráfico, y por su potencial mejor uso del espectro en redes ópticas flexibles, este trabajo se focaliza en proponer un algoritmo RMLSA para redes flexibles de operación dinámica.

\section{ALGORITMOS RMLSA}

La mayoría de los algoritmos RMLSA propuestos para redes ópticas dinámicas y flexibles resuelven el problema de manera secuencial [19]: primero la asignación de la ruta (RA: Routing Allocation), luego la definición del formato y/o nivel de modulación (MLA: Modulation Level Allocation) y, finalmente, la asignación de espectro (SA: Spectrum Allocation).

Los algoritmos de asignación de ruta (RA) más usados en la literatura son del tipo fijo y fijo-alternado [20]. En el ruteo fijo, una tabla de ruteo precalculada mantiene la información de una única ruta por cada posible par de nodos fuente-destino (generalmente, el camino más corto). Así, ante una petición de conexión entre 2 nodos, si la ruta asociada en la tabla de ruteo no tiene suficientes FSUs disponibles o no cumple con todas las restricciones del problema RMLSA mencionadas en la sección anterior, la petición de conexión se rechaza. En cambio, en el ruteo fijoalternado, la tabla de ruteo precalculada mantiene $\mathrm{k}$ rutas (por ejemplo, los k-caminos más cortos). Para cada petición de conexión, se trata de establecer la conexión en alguna de ellas hasta agotar todas las posibilidades (el orden en que se verifican las rutas depende del algoritmo usado). Solo si ninguna de las k posibles rutas puede asignarse, la petición de conexión se rechaza. 
En relación a los algoritmos de asignación de formato y/o nivel de modulación (MLA), el esquema más usado en la literatura es asociar de manera prefijada la velocidad de transmisión digital con un formato de modulación específico [19]. Esta asociación o el par "velocidad de transmisión digital-formato y nivel de modulación" definen el ancho de banda (en términos de FSUs) de una conexión óptica y además, el alcance en $[\mathrm{km}]$. Por lo tanto, se busca establecer una conexión óptica, cuyo largo de la ruta candidata cumpla con el alcance del par velocidad de transmisión digital-formato y/o nivel de modulación. Para la elección de los formatos y/o nivel de modulación, los trabajos previos se han focalizado en asignar un canal con una única portadora modulada (por ejemplo, BPSK, QPSK, 16-QAM, entre otros) o por medio de la construcción de un conjunto de subportadoras moduladas a menor tasa de transmisión digital que usando una portadora única (la suma de la tasa de transmisión de cada subportadora resulta en la tasa de transmisión digital de la conexión óptica completa). La elección del formato y/o nivel de modulación, para sistemas con una única portadora o múltiples subportadoras, se basa en encontrar la opción que minimice el número de FSU's necesarios o que experimente el menor consumo energético con la condición que el alcance de la señal sea mayor o igual a la ruta candidata.

En la siguiente etapa (asignación de espectro, SA), los algoritmos más usados en la literatura son First Fit (primer segmento espectral disponible [7]) y Random Fit (asignación aleatoria [19]). Ante una petición de conexión, donde la ruta es determinada por el algoritmo RA y el ancho de banda (en términos de FSUs) por el algoritmo MLA, Random Fit asigna aleatoriamente un segmento disponible de espectro, lo que produce un alto grado de fragmentación. En cambio, First Fit asigna ordenadamente el espectro, buscando la primera porción suficiente de espectro disponible que pueda contener la demanda, provocando una menor fragmentación del espectro que Random Fit y obteniendo menores probabilidades de bloqueo [21].

En este artículo se propone una solución al problema RMLSA que minimiza el uso de subportadoras OFDM y que asigna el espectro tomando en cuenta la velocidad de transmisión digital de la conexión. Todo esto, con tal que el alcance de la señal conformada para establecer la conexión óptica tenga un alcance igual o mayor al de la longitud de la ruta escogida.

\section{PROPUESTA DE ALGORITMO RMLSA}

El algoritmo RMLSA propuesto se denomina kSPMAS-BRAF ( $k$-Shortest Path - Minimum Allocation of Subcarriers - Bit Rate Aware Fit) y se compone de 3 etapas secuenciales de asignación de recursos: ruta, formato y nivel de modulación, y espectro.

kSP-MAS-BRAF está basado en el uso de OFDM para conformar cada canal óptico que se transmite por una fibra óptica. A diferencia de los formatos de modulación digitales tradicionales, donde se modula una única señal portadora a una gran velocidad de transmisión digital, en OFDM se modulan distintas subportadoras a menores tasas que, en su conjunto, proveen la tasa de bits total de la petición [22] (la velocidad de transmisión digital entre los nodos $s$ y $d, B_{s, d}$, está dado por $B_{s, d}=N_{s c} \cdot B_{s c}$, donde $N_{s c}$ es el número de sub-potadoras y $B_{s c}$ es el bit rate de cada una de ellas). En la Figura 2 se ilustra el uso de OFDM para conformar los canales ópticos de manera flexible. En esta, se puede apreciar el espectro de un enlace óptico ocupado por 2 conexiones OFDM con distintos números de subportadoras.

Una de las restricciones de funcionamiento de OFDM es que las subportadoras deben ser ortogonales entre sí. Para esto, la separación espectral $(\Delta f)$ entre peaks de amplitud de cada subportadora (ver Figura 2) debe ser igual a $1 / T_{s c}$, donde $T_{s c}$ representa la duración de un símbolo [22]. Se tiene, además, que la velocidad de transmisión digital $B_{s c}$ (en bps) de cada subportadora está dada por: $B_{s c}=\lambda /$ $T_{s c}=\lambda \cdot \Delta f$ (donde $\lambda$ representa el número de bits por cada símbolo usado en el formato y nivel de modulación escogido). De esta forma, para asegurar ortogonalidad, se supone que la tasa de símbolos $1 / T_{s c}$ debe tener un ancho de banda equivalente a un

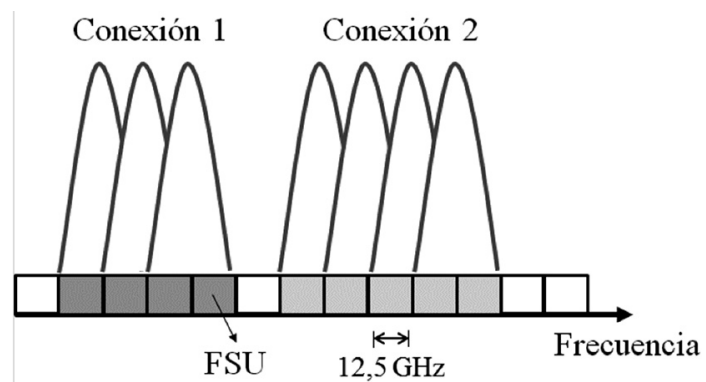

Figura 2. Canales ópticos conformados por subportadoras. 
FSU (habitualmente 12,5 GHz). Así, y de acuerdo con la Figura 2, el número de FSU requeridas por una conexión sería aproximadamente $\left[N_{s c}+1\right]$.

Por otro lado, el alcance de cada subportadora, $A_{s c}$ en $[\mathrm{km}]$ depende del formato y nivel de modulación. Cabe destacar el alcance de la señal OFDM completa es igual al alcance individual de cada subportadora.

Ante una solicitud de conexión entre un par de nodos $s, d$ y a una velocidad de trasmisión digital $B_{s, d}$, kSP-MAS-BRAF ejecuta los pasos descritos en el pseudocódigo de la Figura 3, donde:

- $s$ : Nodo fuente.

- $d$ : Nodo destino.

- $\quad B_{s, d}$ : Velocidad de transmisión digital (bit rate) solicitada entre los nodos $s$ y $d$.

- $\quad k$ : Número de rutas consideradas entre los nodos $s$ al $d$.

- $\Re_{s, d}$ : Conjunto de rutas (tabla de ruteo) entre $\operatorname{los} \operatorname{nodos} s$ al $d . \Re_{s, d}=\left\{R_{s, d}^{1}, R_{s, d}^{2}, \ldots \ldots R_{s, d}^{k}\right\}$

- $\quad R_{s, d}^{i}: i$-ésima ruta más corta entre los nodos $s$ al $d$.

- $\quad M$ : Conjunto de los pares formato y nivel de modulación soportados por cada transpondedor de la red, ordenados de mayor a menor eficiencia espectral. $M=\left\{M^{1}, M^{2}, \ldots ., M^{\|M\|}\right\}$

- $\quad M^{j}: i$-ésimo par formato y nivel de modulación disponible en los transpondedores de la red.

- $\quad\|M\|$ : Cardinalidad de $M$, es decir, el número de pares formato y nivel de modulación soportados por los transpondedores.

- $A_{s c}\left(M^{j}\right)$ : Alcance máximo, en $\mathrm{km}$, de una subportadora OFDM con el par formato y nivel de modulación $M^{j}$.

- $\quad L\left(R_{s, d}^{i}\right)$ : Largo de la ruta $R_{s, d}^{i}$, en km.

- $\quad L_{s, d}$ : Conjunto de los largos de las rutas entre los nodos $s$ al $d$. De esta forma, $L_{s, d}=\left\{L\left(R_{s, d}^{1}\right), L\left(R_{s, d}^{2}\right), \ldots L\left(R_{s, d}^{k}\right)\right\}$.

- $\quad B_{s c}\left(M^{j}\right)$ : Velocidad de transmisión digital de una subportadora OFDM en función del par formato y nivel de modulación $M^{j}$.

- $\quad F S U_{s, d}$ : Número de FSUs asociados a la petición de conexión entre los nodos $s$ y $d$.

- $G B$ : Banda de guarda medida en número de FSUs. Se supone un valor de 1.

- $f$ : Número máximo de FSUs en una banda de operación de una fibra óptica.

- $U^{e}$ : Vector booleano de disponibilidad de FSUs del enlace $e$, el cual está compuesto por

\section{Paso 1: Inicialización de variables: $\boldsymbol{i}=1$}

Paso 2: Algoritmo de ruteo:

$$
\text { - } \quad \text { Obtener } R_{s, d}^{i} \text { desde conjunto } \Re_{s, d} \text {. }
$$

Paso 3: Algoritmo de asignación de formato y nivel de modulación y número de FSUs necesarias:

Paso 3.1: Obtener $L\left(R_{s, d}^{i}\right)$ desde el conjunto $\mathfrak{L}_{s, d}$.

Paso 3.2: $j=1$

While $j \leq\|\mathcal{M}\|$ do

$$
\text { If } L\left(R_{s, d}^{i}\right)<A_{s c}\left(M^{j}\right) \text { then }
$$

- Asignar $M^{j}$ a la petición de conexión entre los nodos $s$ y $d$.

- Pasar al Paso 3.3.

Else

$$
j=j+1,
$$

\section{End While}

$$
\begin{aligned}
& i=i+1 \text {, } \\
& \text { If } i \leq k \text { then } \\
& \text { Else } \\
& \text { - Volver al Paso } 2 . \\
& \text { Rechazar solicitud de conexión. }
\end{aligned}
$$

Paso 3.3: $N=1$,

$$
\begin{aligned}
& \text { While } B_{s, d}>N \cdot B_{s c}\left(M^{j}\right) \\
& \qquad \begin{array}{l}
N=N+1 \\
\text { End While }
\end{array} \\
& \qquad \begin{array}{l}
N_{s c}=N \\
F S U_{s, d}=\left\lceil N_{s c}+1\right\rceil+G B
\end{array}
\end{aligned}
$$

Paso 4: Algoritmo de asignación de espectro.

$$
\text { Paso 4.1: Obtener } U\left(R_{s, d}^{i}\right)
$$

Paso 4.2: Buscar y almacenar todos los bloques de FSUs consecutivos disponibles (índice y tamaño) de $U\left(R_{s, d}^{i}\right)$.

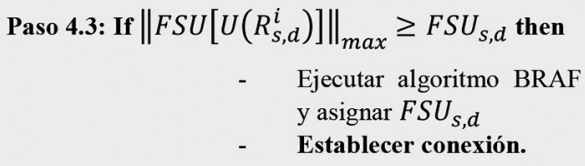

Else

$$
\begin{aligned}
& \quad \begin{array}{l}
i= \\
\text { If } i \leq k \text { then } \\
\quad \text { Volver al Paso } 2 .
\end{array} \\
& \text { Else } \\
& \quad \text { Rechazar solicitud de conexión }
\end{aligned}
$$

Figura 3. Pseudo-código de kSP-MAS-BRAF.

$\left[u_{1}^{e}, u_{2}^{e}, \ldots, u_{i}^{e}, \ldots u_{f}^{e}\right]$, donde $u_{i}^{e}$ representa la disponibilidad del $i$-ésimo FSU del enlace $e$.

- $\quad U\left(R_{s, d}^{i}\right):$ El vector de disponibilidad (compuesto de $f$ elementos) de FSUs de la ruta candidata 
$R_{s, d}^{i}$, el que se obtiene realizando la operación lógica AND entre los vectores de disponibilidad de FSUs de los enlaces, $U^{e}$, que componen la ruta $R_{s, d}^{i}$.

- $\quad\left\|F S U\left[U\left(R_{s, d}^{i}\right)\right]\right\|_{\max }:$ Número mayor de FSUs contiguos en $U\left(R_{s, d}^{i}\right)$.

Las características generales de operación de cada etapa del algoritmo son las siguientes:

En la etapa de asignación de rutas (Paso 2 en la Figura 3), se trabaja con un algoritmo de ruteo fijo-alternado. Es decir, se considera una tabla de ruteo prefijada en la que cada conexión tiene asociadas $k$ rutas candidatas.

En la etapa de asignación de formato y nivel de modulación (Paso 3 en la Figura 3), el algoritmo denominado Asignación Mínima de Subportadoras o MAS (Minimum Allocation of Subcarriers) se basa en el uso de OFDM y asigna el formato y nivel de modulación (a diferencia de propuestas que solo elijen el nivel de modulación, dejando fijo el formato de modulación [23]) que menor ancho de banda requiere con tal de cumplir con un alcance óptico suficiente para cubrir la longitud de la ruta.

En la etapa de asignación de espectro (Paso 4 en la Figura 3), se aplica un algoritmo SA propuesto por los autores de este artículo, en [21]. El algoritmo, denominado BRAF (Bit Rate Aware Fit), toma en consideración la tasa de transmisión digital de las peticiones de conexión para asignar el espectro. Para ello, BRAF divide el espectro en dos zonas: las conexiones que requieren bajas tasas de transmisión se asignan al espectro usando First Fit desde el menor al mayor identificador de FSU y las conexiones con velocidades de transmisión altas se asignan al espectro usando First Fit desde el mayor al menor identificador de FSU (o equivalente a Last Fit). Se asigna el primer bloque de FSUs que se encuentre disponible y que soporte a la demanda. Este algoritmo de asignación de espectro reduce notoriamente la fragmentación del espectro [21] y, como consecuencia, la probabilidad de bloqueo, respecto de Random y First Fit.

\section{RESULTADOS}

El rendimiento del algoritmo propuesto se evaluó en términos de la probabilidad de bloqueo (PB) mediante la implementación de un simulador de eventos discretos en lenguaje $\mathrm{C}++$. Este simulador recrea el proceso de establecimiento de las conexiones y la posterior liberación de recursos cuando estas finalizan la transmisión de datos.

La probabilidad de bloqueo (PB) se puede estimar dividiendo el número de peticiones conexión rechazadas por el número total de peticiones de conexión (rechazadas más las establecidas).

La topología usada fue la NSFNet (National Science Foundation's Network), de 14 nodos y 21 cables, que se muestra en la Figura 4 junto con las longitudes de los enlaces en [km]. Se supone que cada cable está compuesto por 2 enlaces unidireccionales o fibras ópticas. Se supone que cada fibra óptica tiene un ancho de banda de $4.000 \mathrm{GHz}$ centrados en $193,1 \mathrm{THz}$ (correspondiente a la banda C) y que cada FSU tiene un ancho de banda de $12,5 \mathrm{GHz}$, lo que resulta en un ancho de banda total de cada fibra óptica igual a 320 FSUs.

Cada conexión, definida por un par de nodos fuente-destino, genera tráfico del tipo ON-OFF. La duración de ambos períodos (ON y OFF) se modela con una variable aleatoria exponencial. $\mathrm{La}$ duración media del periodo ON (OFF) se denota por $t_{O N}\left(t_{O F F}\right)$. De esta forma, la carga de tráfico ofrecida por cada conexión a la red $(p)$, está dada por la expresión: $t_{O N} /\left(t_{O N}+t_{O F F}\right)$. En este artículo, se supone que la carga de tráfico $p$ es la misma para todas las conexiones. El valor supuesto para $t_{O N}$ es de 0,1 [s] y el valor de $t_{O F F}$ se ajusta para generar cargas de tráfico $(p)$ de 0,1 a 0,9 .

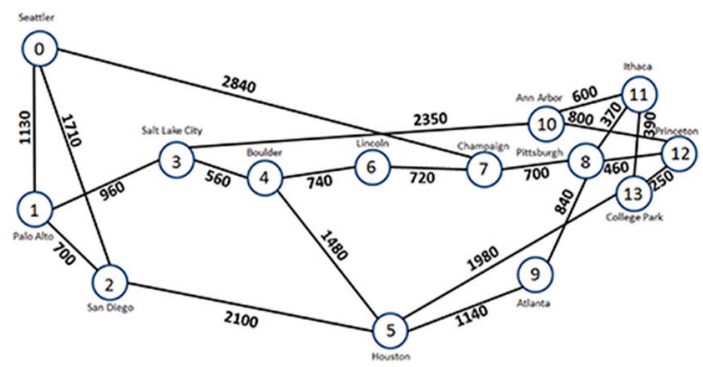

Figura 4. Topología de red NSFNet.

La Tabla 1 muestra los formatos y niveles de modulación, capacidad y alcance (sin regeneración) de las subportadoras OFDM considerados en este artículo [21]. Debido a que el mayor alcance es 
de $4.000 \mathrm{~km}$, las longitudes de los enlaces de la NSFNet (Figura 4) se reducen al 40\%.

Las velocidades de transmisión digitales soportadas por cada transpondedor de la red son: 10, 40,100, 400 y 1.000 Gbps. La distribución de probabilidad de las solicitudes de velocidades de transmisión se supone uniforme.

Tabla 1. Formatos y niveles de modulación, capacidad y alcance de subportadoras OFDM [15].

\begin{tabular}{|c|c|c|c|}
\hline $\begin{array}{c}\text { Formato } \\
\text { y nivel de } \\
\text { modulación } \\
M^{j}\end{array}$ & $\begin{array}{c}\text { Bits por } \\
\text { símbolo } \\
\lambda\end{array}$ & $\begin{array}{c}\text { Bit rate de } \\
\text { subportadora } \\
B_{s c}\left(M^{j}\right) \text { en } \\
{[G b p s]}\end{array}$ & $\begin{array}{l}\text { Alcance } \\
\text { máximo } \\
A_{s c}\left(M^{j}\right) \\
\text { en }[\mathrm{km}]\end{array}$ \\
\hline 64QAM & 6 & 75 & 125 \\
\hline 32QAM & 5 & 62,5 & 250 \\
\hline 16QAM & 4 & 50 & 500 \\
\hline 8QAM & 3 & 37,5 & 1.000 \\
\hline QPSK & 2 & 25 & 2.000 \\
\hline BPSK & 1 & 12,5 & 4.000 \\
\hline
\end{tabular}

El algoritmo de ruteo en kSP-MAS-BRAF ( $k$-Shortest Path - Minimum Allocation of Subcarriers Bit Rate Aware Fit), utiliza $k=3$ (3 rutas candidatas más cortas por cada conexión). Para el algoritmo de asignación de espectro BRAF [21], las peticiones de conexión de 400 y 1.000 Gbps se consideran de alta velocidad de transmisión (o sea, se usa el algoritmo de asignación de espectro Last Fit para conexiones con estos bit rates) y el resto, las trata como de baja velocidad (o sea, se usa el algoritmo de asignación de espectro First Fit) [17, 18, 21].

La Figura 5 muestra la probabilidad de bloqueo de la propuesta 3SP-MAS-BRAF (triángulo rojo) junto con la de otros 5 algoritmos RMLSA en los que solo se varían el algoritmo MLA (Modulation Level Assignment) y SA (Spectrum Assignment): MAS-FF (FF: First Fit) con triángulo azul, MAS-RF (RF: Random Fit) con triángulo gris, RMOD-FF (círculo rojo), RMOD-FF (círculo azul), y RMODRF (triángulo gris). RMOD (Random Modulation) representa un algoritmo de asignación de formatos de modulación que asigna aleatoriamente alguno de los formatos de modulación que cumplen con el requisito de que el alcance óptico es mayor a la longitud de la ruta.
Se puede apreciar que el algoritmo propuesto obtiene la menor probabilidad de bloqueo para toda carga de tráfico. En el mejor caso, obtiene un rendimiento superior en más de un orden de magnitud con el de peor desempeño (3SP-RMOD-RF). Se observa también que los algoritmos basados en MAS tienen mejor rendimiento que los basados en RMOD debido a su mayor eficiencia en el uso del espectro. Esto destaca el hecho de que una buena elección de un formato y nivel de modulación es clave para el uso eficiente de recursos, ya que dejando de lado los algoritmos que usan RF para asignar espectro, se puede observar que el factor determinante en el rendimiento es la elección del algoritmo de asignación de formato y nivel de modulación. En lo que respecta a asignación de espectro, los algoritmos que usan BRAF obtienen un desempeño solo levemente superior a FF.

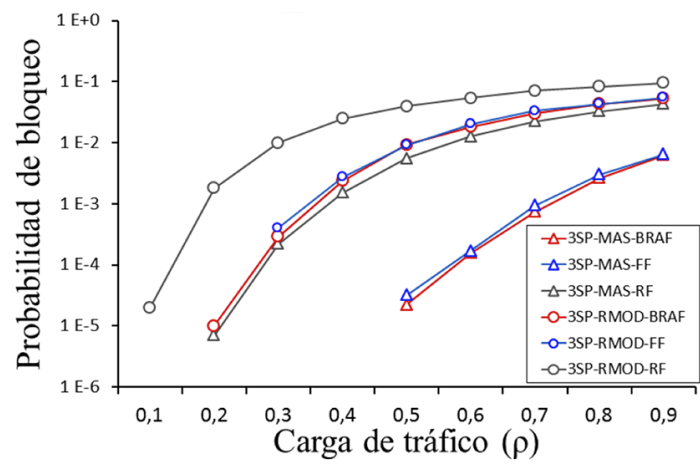

Figura 5. Probabilidad de bloqueo en función de la carga de tráfico para 6 algoritmos RMLSA.

A continuación, en la Figura 6 se muestra el índice de fragmentación promedio de espectro de la red $\left(I F_{\text {red }}\right)$ aplicado a los algoritmos evaluados (la distribución de símbolos y colores identificadores de cada algoritmo son los mismos que los usados en la Figura 5). Este índice consiste en la cuantificación del número promedio de FSUs disponibles y consecutivos presentes en todos los enlaces de fibra óptica de la red en un instante determinado, el que se pondera por el tiempo en que se encuentra en este estado. Los detalles de este indicador pueden obtenerse en el artículo publicado por los mismos autores de esta propuesta en [21]. El índice de fragmentación puede tomar valores entre 0 y 1 . El valor 0 se obtiene cuando todos los FSUs se 
encuentran disponibles y, por ende, no existe fragmentación. En contraste, el valor 1 se obtiene cuando no existen FSUs disponibles. El índice de fragmentación es más cercano a 1 mientras mayor es el nivel de fragmentación del espectro disponible.

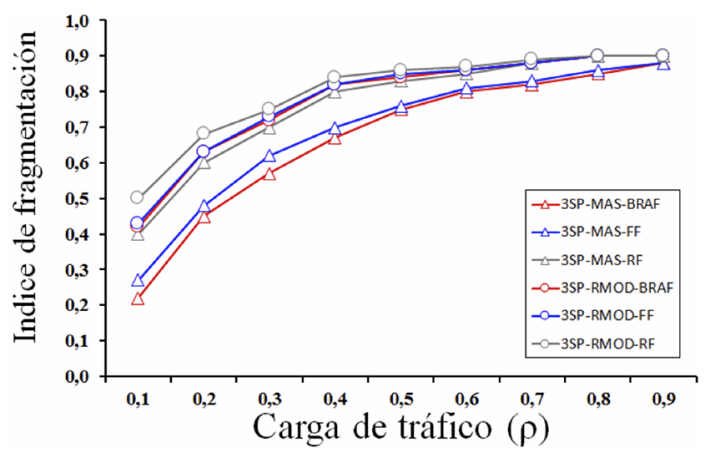

Figura 6. Índice de fragmentación de la red en función de la carga de tráfico para los algoritmos RMLSA analizados.

De la Figura 6 se puede apreciar que el mejor rendimiento en términos de índice de fragmentación lo obtienen los algoritmos RMLSA con la asignación de formato y nivel de modulación MAS (Minimum Allocation of Subcarriers) por sobre todos los algoritmos basados en RMOD (Random Modulation), incluso para el algoritmo de asignación espectro de peor desempeño (Random Fit). De esta forma, se aprecia la incidencia del algoritmo de asignación de formato y nivel de modulación dentro del rendimiento del algoritmo RMLSA.

Por otro lado, y también apreciado de la Figura 6, el algoritmo de asignación BRAF (Bit Rate Aware Fit) obtiene valores menores de fragmentación que FF (First Fit) y RF (Random Fit), coincidiendo con lo obtenido en [21].

\section{CONCLUSIONES}

En este artículo se ha presentado una propuesta de solución al problema RMLSA en redes ópticas dinámicas flexibles que operan con OFDM. El rendimiento de la propuesta, denominada kSP-MASBRAF, se comparó mediante simulación con el de otras alternativas usadas en la literatura. Los resultados obtenidos muestran que la propuesta obtiene menores valores de probabilidad de bloqueo en todo el rango de cargas de tráfico, incluso hasta en un orden de magnitud. Además, se comprueba el impacto en el rendimiento del uso algoritmos de asignación de formatos y niveles de modulación dentro de RMLSA.

\section{AGRADECIMIENTOS}

$\mathrm{Al}$ apoyo financiero de los proyectos FONDECYT 1130388 y 11140386 , PIA de la DI-PUCV 037.467/2015, y DGIP USM 23.12.29.

\section{REFERENCIAS}

[1] J.M. Simmons, "Optical network design and planning", Springer, 2nd edition, New Jersey, USA, ISBN: 3319052268, 2014. ITU-T Recommendation G.694.1, "Spectral grids for WDM applications: DWDM frequency grid (2012)", 2012.

[3] ITU-T Recommendation G.709, "Interfaces for the Optical Transport Network (OTN)", 2012.

[4] J. Sakaguchi, B.J. Puttnam, W. Klaus, Y. Awaji, N. Wada, A. Kanno, T. Kawanishi, K. Imamura, H. Inaba, K. Mukasa, R. Sugizaki, T. Kobayashi, M. Watanabe, “305 Tb/s Space Division Multiplexed Transmission Using Homogeneous 19-Core Fiber", Journal of Lightwave Technology, vol. 31, $\mathrm{N}^{\mathrm{o}} 4$, pp. 554-562, February 2013. ISSN: 07338724, DOI: 10.1109/JLT.2012.2217373.

[5] A. Napoli, M. Bohn, D. Rafique, A. Stavdas, N. Sambo, L. Potì, M. Nölle, J.K. Fischer, E. Riccardi, A. Pagano, A. Di Giglio, M. Svaluto, J.M. Fabrega, E. Hugues-Salas, G. Zervas, D. Simeonidou, P. Layec, A. D'Errico, T. Rahman, J.P. Fernández-Palacios Giménez, "Next Generation Elastic Optical Networks: The Vision of the European Research Project IDEALIST", IEEE Communications Magazine, vol. 53, $\mathrm{N}^{\mathrm{o}}$ 2, pp. 152-162, February 2015. ISSN: 0163-6804, DOI: 10.1109/MCOM.2015.7045404.

[6] M. Jinno, H.Takara, Y. Sone, K. Yonenaga, and A. Hirano, "Elastic optical path network architecture: Framework for spectrallyefficient and scalable future optical networks", IEICE Transaction on Commun. E95-B, pp. 706-713, March 2012. ISSN: 1745-1345, DOI: 10.1587/transcom.E95.B.706.

[7] O. Gerstel, M. Jinno, A. Lord, and S.J.B. Yoo, "Elastic optical networking: a new dawn 
for the optical layer?", Communications Magazine IEEE, vol. 50, No 2, pp. s12 - s20, February 2012. ISSN: 0163-6804, DOI: 10.1109/MCOM.2012. 6146481.

[8] T. Morioka, M. Jinno, H. Takara, and H. Kubota, "Innovative Future Optical Transport Network Technologies", NTT Technical Review, pp. 1-11. August 2011, March 2016: www.ntt-review.jp

[9] M. Jinno, T. Hidehiko, and K. Bartlomiej, "Spectrum-efficient and scalable elastic optical path network: architecture, benefits, and enabling technologies", IEEE Communications Magazine, vol. 47, $\mathrm{N}^{\mathrm{o}} 11$, pp. 66-73, November 2009. ISSN: 0163-6804, DOI: 10.1109/MCOM.2009. 5307468.

[10] S. Shakya, Xiaojun Cao, Zilong Ye, Chunming Qiao, "Spectrum allocation in spectrum-sliced elastic optical path networks using traffic prediction", Photonic Network Communications, vol. 30, $\mathrm{N}^{\circ} 1$, pp. 131142, August 2015. ISSN: 1387-974X, DOI: 10.1007/s11107-015-0489-z.

[11] L. Velasco, M. Klinkowski, M. Ruiz, J. Comellas, "Modeling the Routing and Spectrum Allocation Problem for Flexgrid Optical Networks", Photonic Network Communications, vol. 24, $\mathrm{N}^{\circ} 3$, pp. 177-186, December 2012, ISSN: 1572-8188, DOI: 10.1007/s11107-012-0378-7.

[12] A. Alyatama, "Computing the single-link performance measurement for elastic optical OFDM", Photonic Network Communications, Available online: March 2016. ISSN: 15728188, DOI: 10.1007/s11107-016-0624-5.

[13] I. Tomkos, "A survey of recent developments on flexible/elastic optical networking", 14th International Conference on Transparent Optical Networks (ICTON), Coventry, England, July 2012.

[14] Y. Yin, K. Wen, D. Geisler, R. Liu, and S. J. B. Yoo, "Dynamic on-demand defragmentation in flexible bandwidth elastic optical networks", Optics Express, vol. 20, No 2, pp. 1798-1804, January 2012. ISSN: 10944087, DOI: 10.1364/OE.20. 001798.

[15] K. Cristodoulopoulos, I. Tomkos, and E. Varvarigos, "Spectrally/Bitrate Flexible Optical Network Planning". 36th European Conference and Exhibition on Optical Communication (ECOC), Torino, Italy, September 2010.
[16] Y. Wang, X. Cao, and Y. Pan, "A study of the routing and spectrum allocation in spectrumsliced elastic optical path networks", 30th IEEE International Conference on Computer Communications (INFOCOM), Shangai, China, April 2011.

[17] Y. Wang, X. Cao, and Q. Hu, "Routing and Spectrum Allocation in Spectrum-Sliced Elastic Optical Path Networks", IEEE International Conference on Communications (ICC), Kyoto, Japan, June 2011.

[18] F.S. Abkenar, A.G. Rahbar, "Study and Analysis of Routing and Spectrum Allocation (RSA) and Routing, Modulation and Spectrum Allocation (RMSA) Algorithms in Elastic Optical Networks (EONs)", Optical Switching and Networking, Vol. 23, $\mathrm{N}^{\mathrm{o}} 1$, pp. 5-39, January 2017. ISSN: 1573-4277, DOI: 10.1016/j.osn.2016.08.003

[19] B.C. Chatterjee, N. Sarma, E. Oki, "Routing and Spectrum Allocation in Elastic Optical Networks: A Tutorial", IEEE Communications Surveys \& Tutorials, vol. 17, No 3, pp. 17761800, August 2015. ISSN: 1553-877X, DOI: 10.1109/COMST. 2015.2431731.

[20] H. Zang, J.P. Jue, and B. Mukherjee, "A Review of Routing and Wavelength Assignment Approaches for WavelengthRouted Optical WDM Networks", Optical Networks Magazine, pp. 47-60, January 2000. ISSN: 1572-8161 DOI: 10.1.1.127. 5171.

[21] R. Ahumada, A. Leiva, F. Alonso, S. Fingerhuth, and G. Farías, "Spectrum Allocation Algorithms for Elastic DWDM Networks on Dynamic Operation", IEEE Latin America Transactions, vol. 12, No 6, pp. 1012-1018, September 2014. ISSN: 15480992, DOI: 10.1109/TLA.2014. 6893994.

[22] López Vizcaíno, J.; Ye, Y.; López, V.; Jiménez, F.; Duque, R.; Krummrich, P., "Cost Evaluation for Flexible-Grid Optical Networks", IEEE Global Communications Conference (GLOBECOM), Anaheim, CA, USA, December 2012.

[23] X. Wan, N. Hua, and X. Zheng, "Dynamic Routing and Spectrum Assignment in Spectrum-Flexible Transparent Optical Networks", IEEE/OSA Journal of Optical Communications and Networking, vol. 4, $\mathrm{N}^{\circ} 8$, pp. 603.613, August 2012, ISSN: 1943-0620, DOI: 10.1364/JOCN.4.000603 